\title{
N-Acetylcysteine Normalizes Glutamate Levels in Cocaine-Dependent Patients: A Randomized Crossover Magnetic Resonance Spectroscopy Study
}

\author{
Lianne Schmaal*,', Dick J Veltman ${ }^{1,2}$, Aart Nederveen ${ }^{3}$, Wim van den Brink' and Anna E Goudriaan' \\ 'Amsterdam Institute for Addiction Research, Department of Psychiatry, Academic Medical Center, University of Amsterdam, Amsterdam, \\ The Netherlands; ${ }^{2}$ Department of Psychiatry, VU University Medical Center, Amsterdam, The Netherlands; ${ }^{3}$ Department of Radiology, Academic \\ Medical Center, University of Amsterdam, Amsterdam, The Netherlands
}

\begin{abstract}
Treatment with $\mathrm{N}$-acetylcysteine (NAC) normalizes glutamate (Glu) homeostasis and prevents relapse in drug-dependent animals. However, the effect of NAC on brain Glu levels in substance-dependent humans has not yet been investigated. Proton magnetic resonance spectroscopy ('H MRS) was used to investigate Glu changes in the dorsal anterior cingulate cortex (dACC) after a single dose of NAC in cocaine-dependent patients and normal controls. In an open-label, randomized, crossover study, 8 cocaine-dependent patients and 14 healthy controls underwent two scan sessions: one group receiving no compound and the other following a single administration of $2400 \mathrm{mg}$ NAC. The Barratt Impulsiveness Scale was administered to examine the relation between dACC Glu levels and impulsivity. In the medication-free condition, Glu levels in the dACC were significantly higher in cocaine-dependent patients compared with healthy controls. After administration of NAC, Glu levels were reduced in the cocaine-dependent group, whereas NAC had no effect in healthy controls. Higher baseline Glu levels were associated with higher impulsivity, and both were predictive of greater NAC-induced Glu reduction. The current findings indicate that NAC can normalize elevated Glu levels in cocaine-dependent patients. These findings may have important implications for treatment, because abnormal Glu levels are related to relapse, and treatment with NAC prevented relapse in animal studies. Furthermore, clinical studies have indicated beneficial effects of NAC in cocaine-dependent patients, and the current study suggests that these beneficial effects might in part be mediated by the ability of NAC to normalize glutamatergic abnormalities.

Neuropsychopharmacology (2012) 37, 2I43-2152; doi:I0.1038/npp.2012.66; published online 2 May 2012
\end{abstract}

Keywords: cocaine dependence; $\mathrm{N}$-acetylcysteine; glutamate; magnetic resonance spectroscopy; impulsivity

\section{INTRODUCTION}

Traditionally, research into the neurobiological substrate of drug addiction has focused on mesolimbic dopamine reward circuitry. However, recent literature has highlighted the importance of the excitatory amino-acid glutamate (Glu) in cocaine dependence, especially its role in the continuation of and relapse into substance abuse. In rodents, protracted cocaine use induces neural changes in glutamatergic signaling, resembling neuroplasticity associated with learning and memory (Kelley, 2004). Specifically, repeated cocaine exposure has been found to result in

*Correspondence: L Schmaal, Amsterdam Institute for Addiction Research, Department of Psychiatry, Academic Medical Center, University of Amsterdam, P.O. Box 22660 II00 DD Amsterdam, The Netherlands, Tel: +3120 8913762, Fax: + 3120 8913701,

E-mail: lianschmaal@gmail.com

Received 3 November 201 I; revised 16 March 2012; accepted 5 April 2012 reduced firing rates of glutamatergic projections from the medial prefrontal cortex (including the anterior cingulate cortex (ACC)) to the nucleus accumbens (Sun and Rebec, 2006) and reduced levels of extracellular Glu in the nucleus accumbens under basal conditions (Baker et al, 2003a). In the presence of cocaine-related cues, a large increase of synaptic Glu release derived from prefrontal afferents has been observed in the nucleus accumbens, in part resulting from reduced tone of extracellular Glu on group II metabotropic Glu (mGluR2/3) receptors that are important for regulating synaptic Glu release (Baker et al, 2003a; McFarland et al, 2003; Sun and Rebec, 2006). These neuroadaptations may be of key importance for cocaine reinstatement in self-administration animal models of relapse (Baker et al, 2003a; Madayag et al, 2007; McFarland et al, 2003; Sun and Rebec, 2006) and have led to the suggestion that targeting the glutamatergic system may prove effective when treating cocaine dependence (Reissner and Kalivas, 2010). A potential glutamatergic drug for 
treating substance abuse is $N$-acetylcysteine (NAC), an amino-acid cystine prodrug, which is used to treat acetaminophen overdose and sold over the counter as a mucolytic agent and nutritional supplement. Systemic administration of NAC restores extracellular Glu levels (thereby increasing tonic activation of the mGluR2/3 receptors) and prevents relapse to drug-seeking behavior in rats previously treated with cocaine (Baker et al, 2003b; Madayag et al, 2007) and heroin (Zhou and Kalivas, 2008). In humans, pilot studies have shown that NAC decreases cue-induced craving for cocaine (LaRowe et al, 2007), pathological gambling (Grant et al, 2007), number of cigarettes smoked (Knackstedt et al, 2009), the rewarding effect of smoking (Schmaal et al, 2011), and marijuana use and craving (Gray et al, 2010). However, whether these beneficial effects of NAC are mediated by NAC-induced Glu changes in the human brain has not yet been investigated.

A technique that allows in vivo assessment of Glu levels, along with other neurometabolite levels in the human brain, is proton magnetic resonance spectroscopy ( ${ }^{1} \mathrm{H}$ MRS). A few studies have used ${ }^{1} \mathrm{H}$ MRS to examine Glu in substance dependence. Decreased levels of Glu have been found in the rostral ACC (rACC) in cocaine-dependent patients (Yang et al, 2009), but both increased (Lee et al, 2007) and decreased (Thoma et al, 2011) Glu levels were found in the dorsal ACC in alcohol-dependent patients. In addition, increased Glu levels were found in the putamen in squirrel monkeys that were treated with cocaine for 9 months (Liu et al, 2011), although no abnormalities were found in chronic tobacco smokers (Gallinat and Schubert, 2007). With regard to the combined Glu plus glutamine signal, decreased levels have been found in the dorsal ACC in opiate addiction (Yucel et al, 2007). These inconsistent findings could be related to differences in patients' drug use characteristics such as time of abstinence and drug intake between the studies, as Glu abnormalities seem to be highly dependent on individual drug use characteristics (Chang et al, 1997; Ernst and Chang, 2008; Lee et al, 2007; Liu et al, 2011; Yang et al, 2009). Given these findings of Glu abnormalities in substance-dependent patients, it is important to establish whether NAC can normalize Glu alterations observed in substance-dependent individuals. Therefore, the current pilot study aimed to investigate the effect of a single dose of NAC $(2400 \mathrm{mg})$ on brain Glu levels in cocaine-dependent human subjects relative to healthy controls using ${ }^{1} \mathrm{H}$ MRS. Pretreatment with a single dose of systemically administered NAC has been shown to prevent cocaine-primed reinstatement in animals (Moran et al, 2005). This effect was blocked by coadministration of a mGluR2/3 antagonist, indicating that this prevention of reinstatement by a single dose of NAC resulted from modulation of the Glu system (Moran et al, 2005). We chose the dorsal ACC (dACC) as our region of interest because most of the Glu abnormalities in previous human studies were located in the ACC, and to ensure that the ${ }^{1} \mathrm{H}$ MRS data were collected from a homogeneous tissue region that contains predominantly gray matter. Dorsal ACC dysfunction plays a key role in cocaine dependence and has been related to impaired impulse inhibition (for a review, see Garavan and Hester, 2007). For example, using functional MRI, it has been shown that decreased activation of the left dACC, as observed in cocaine-dependent patients during response inhibition tasks, is associated with increased impulsive responding (Hester and Garavan, 2004; Li et al, 2008). Moreover, dACC hypoactivations have been reported in response to cocaine-related cues, a finding that has been interpreted as diminished functioning of the brain's 'control network' following cue exposure (Goldstein et al, 2009; Volkow et al, 2011). Recently, a ${ }^{1} \mathrm{H}$ MRS study in subjects with borderline personality disorder demonstrated a positive association between dACC Glu levels and impulsivity (Hoerst et al, 2010). Therefore, we also included a selfreport impulsivity questionnaire to investigate the relation between dACC Glu levels and impulsivity at baseline.

\section{PATIENTS AND METHODS}

\section{Subjects}

Ten male patients currently treated primarily for cocaine dependence (meeting DSM-IV criteria for cocaine dependence; American Psychiatry Association, 1994) were recruited from regional addiction treatment centers (CD group). Fourteen nonsmoking healthy control subjects (HC group) matched on age, sex, and education were included. Exclusion criteria were: substance use disorders (other than cocaine, alcohol, and nicotine for the CD group); current DSM-IV diagnosis (except for attention deficit hyperactivity disorder (ADHD) and antisocial personality disorder in the CD group); lifetime history of head injury with loss of consciousness for $>5 \mathrm{~min}$; neurological disorders; unstable medical condition; low level of education (drop-out before the age of 16 years); any use of medication affecting the central nervous system; MRI ineligibility due to nonremovable metal objects or claustrophobia. Recent drug and alcohol use was assessed with urine tests. All subjects gave written informed consent to participate in this study, which was approved by the Medical Ethical Committee of the Academic Medical Center.

\section{Clinical Assessments}

All subjects were screened for the presence of Axis I psychiatric disorders using the Mini International Neuropsychiatric Interview plus (MINI-plus; Sheehan et al, 1998). General intelligence (IQ) was assessed using the Dutch version of the National Adult Reading Test (NART; Schmand et al, 1991). Alcohol and drug consumption during the preceding 6 months was quantified using the Time Line Follow Back method (TLFB; Sobell and Sobell, 1992). The Fagerström Test for Nicotine Dependence (FTND; Heatherton et al, 1991) was administered to measure level of nicotine dependence. In addition, the Alcohol Use Disorder Identification Test (AUDIT), a 10item questionnaire, was used to identify harmful patterns of alcohol consumption (Babor et al, 1989).

The Barratt Impulsiveness Scale (BIS-11; Patton et al, 1995) was administered at the start of the first session (in case of the NAC condition before medication was taken) to assess self-reported impulsivity. The BIS-11 is a 30 -item questionnaire designed to assess general impulsiveness. Each item is scored on a 4-point scale (rarely/never, occasionally, often, almost always/always), with higher scores indicative of greater impulsivity. Total score as well 
as scores on the cognitive, motor, and nonplanning subscales were assessed for the current study.

\section{Pharmacologic Intervention}

In an open-label, randomized, crossover design, subjects participated in two test sessions separated by 1 to 2 weeks. Before the ${ }^{1} \mathrm{H}$ MRS test sessions, subjects received either a single dose of $2400 \mathrm{mg}$ NAC or no compound. The selection of the $2400 \mathrm{mg}$ dose was based on previous studies showing beneficial effects of 1200 and $2400 \mathrm{mg} /$ day NAC on treatment retention and drug use in cocaine and nicotine dependence (Mardikian et al, 2007; Knackstedt et al, 2009). NAC was administered $1 \mathrm{~h}$ before the ${ }^{1} \mathrm{H}$ MRS scan because the peak plasma concentration of NAC occurs $\sim 1-2 \mathrm{~h}$ after ingestion (Holdiness, 1991).

\section{Magnetic Resonance Spectroscopy Acquisition and Processing}

MRI and MRS data were obtained using a 3.0T Intera MRI scanner (Philips Healthcare, Best, The Netherlands) equipped with a SENSE eight-channel receiver head coil. Three-dimensional T1-weighted images were collected in the sagittal plane using a gradient echo sequence $(\mathrm{TR}=9 \mathrm{~ms}$; $\mathrm{TE}=3.5 \mathrm{~ms} ; 170$ slices; voxel size $1 \times 1 \times 1 \mathrm{~mm}$; matrix size $256 \times 256$ ). Using these images, a single ${ }^{1} \mathrm{H}$ MRS voxel was placed in the left supracallosal ACC (see Figure 1). Voxel placement was done unilaterally to ensure that the ${ }^{1} \mathrm{H}$ MRS data were collected from a homogeneous tissue region that contained predominantly gray matter. The left dACC was chosen on the basis of studies showing left ACC dysfunction in cocaine-dependent patients compared with healthy controls during impulsivity tasks (Hester and Garavan, 2004; Li et al, 2008). MRS was performed using a short-echo point resolved spectroscopy sequence (PRESS; $\mathrm{TR}=2000 \mathrm{~ms}$; voxel size $50 \times 16 \times 10 \mathrm{~mm}$; NEX =64) with a TE of $38 \mathrm{~ms}$. A TE of $38 \mathrm{~ms}$ was chosen because reliable estimates of the Glu signal with this echo time were obtained previously in our lab and it approximates the echo time reported in a study that found improved detection of Glu with a TE of $40 \mathrm{~ms}$ (Mullins et al, 2008). Spectra were acquired using first-order iterative shimming and water suppression was automatically performed by the scanner.
Spectra derived from ${ }^{1} \mathrm{H}$ MRS from 4.0 to 0.2 p.p.m. were analyzed using LCModel (Linear Combination of Model spectra; Provencher, 1993). LCModel is a user-independent analysis method that estimates metabolite levels by fitting the in vivo spectra to a set of previously acquired in vitro spectra (the basis set). LCModel software provides specific basis sets for different scanners, field strengths, and echo times (Provencher, 1993). For the current study, the basis set for a Philips 3T MRI scanner was used. Results are presented in institutional units approximating millimolar level. Spectra of all subjects passed the quality control. We used the Cramér-Rao lower bounds (CRLBs), a measure of the reliability of the fit, $<20 \%$ for each individual peak as the quality criterion (Provencher, 1993). The CRLBs for Glu in all subjects were between 7 and $12 \%$. Additional indicators for quality of the spectra were mean (SD) signal-to-noise ratio and the mean (SD) full width half maximum (FWHM). In the medication-free condition, the signal-to-noise ratio was 16.64 (2.53) and 17.10 (1.85), and had a FWHM of 0.05 (0.02) p.p.m. and 0.05 (0.01) p.p.m. for the $\mathrm{HC}$ and the $\mathrm{CD}$ group, respectively. In the NAC condition, the signal-to-noise ratio was 16.29 (2.23) in the HC group and 16.60 (1.71) in the CD group, and had a FWHM of 0.05 (0.01) p.p.m. for both groups. LCModel estimates both metabolite concentrations referenced to the unsuppressed water signal and concentration ratios (referenced to creatine). Because concentration ratios are less sensitive to relaxation and partial volumes effects than concentrations referenced to the unsuppressed water signal, the ratios of levels of Glu, glutamate + glutamine (Glx), and $\mathrm{N}$-acetylaspartate (NAA) to creatine plus phosphocreatine (Cr) were used in statistical analyses. To ensure that NACinduced changes observed in concentration ratios were not caused by an effect of NAC on creatine, creatine concentrations referenced to the unsuppressed water signal were used to obtain an indication of NAC effects on creatine.

Brain morphology was assessed using a Voxel-Based Morphometry toolbox (VBM8; http://dbm.neuro.uni-jena.de/vbm/) with default settings. The VBM8 toolbox is an extension of the unified segmentation model (Ashburner and Friston, 2005) in which structural images are bias corrected, segmented into gray matter, white matter, and cerebrospinal fluid, and registered combined within the same model. The proportion of gray matter, white matter, and cerebrospinal fluid within the anatomical mask of the
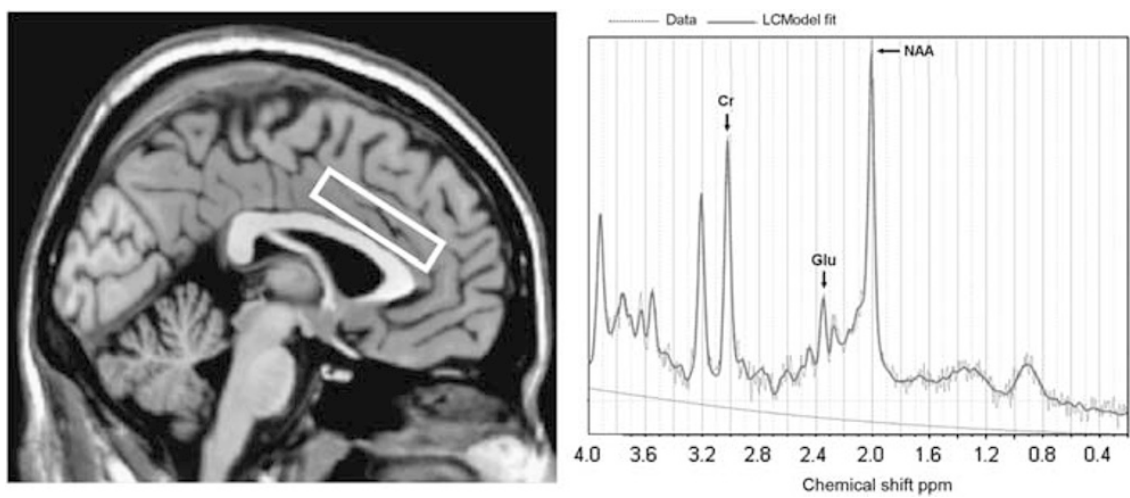

Figure I Voxel placement in left dorsal anterior cingulate cortex for localized single-voxel 'H MRS and a representative spectrum in a healthy control subject. Chemical shift is indicated in parts per million (p.p.m.). Cr, creatine; Glu, glutamate; NAA, N-acetylaspartate. 
Table I Demographic and Clinical Characteristics

\begin{tabular}{|c|c|c|c|c|c|c|}
\hline \multirow[t]{2}{*}{$\begin{array}{l}\text { Demographic } \\
\text { variable }\end{array}$} & \multicolumn{2}{|c|}{$\begin{array}{c}\text { Cocaine- } \\
\text { dependent } \\
\text { group }(N=8)\end{array}$} & \multicolumn{2}{|c|}{$\begin{array}{c}\text { Healthy } \\
\text { control group } \\
(N=14)\end{array}$} & \multirow[t]{2}{*}{$t(d f)$} & \multirow[t]{2}{*}{$P$-value } \\
\hline & Mean & SE & Mean & SE & & \\
\hline Age & 35.12 & 2.42 & 35.71 & 2.44 & $-0.16(20)$ & 0.88 \\
\hline Education (ISCED) & 3.50 & 0.50 & 3.93 & 0.25 & $-0.87(20)$ & 0.40 \\
\hline IQ (NART) & 93.38 & 2.03 & 97.29 & 3.81 & $-0.74(20)$ & 0.47 \\
\hline \multicolumn{7}{|l|}{$B|S-1|$} \\
\hline Total score & 72.75 & 1.81 & 57.50 & 2.09 & $4.92(20)$ & $<0.01$ \\
\hline Cognitive scale & 19.38 & 1.09 & 14.64 & 0.73 & $3.74(20)$ & $<0.01$ \\
\hline Motor scale & 26.12 & 0.76 & 21.14 & 0.90 & $3.74(20)$ & $<0.01$ \\
\hline Nonplanning scale & 27.25 & 0.84 & 21.71 & 0.89 & $4.14(20)$ & $<0.0$ । \\
\hline $\begin{array}{l}\text { Cocaine use in } \\
\text { preceding } 6 \text { months } \\
\text { (in g) }\end{array}$ & 78.69 & 17.27 & 0 & NA & - & - \\
\hline $\begin{array}{l}\text { Alcohol in preceding } \\
6 \text { months (in standard } \\
\text { units) }\end{array}$ & 777.94 & 186.82 & 102.21 & 27.17 & $4.68(7.30)$ & 0.01 \\
\hline AUDIT scores & 21.29 & 3.87 & 4.71 & 0.66 & $4.22(6.35)$ & 0.01 \\
\hline $\begin{array}{l}\text { Number of smokers, } \\
N(\%)\end{array}$ & $\begin{array}{c}7 \\
(87.5)\end{array}$ & NA & 0 & NA & - & - \\
\hline Cigarettes/day & 17.25 & 3.49 & 0 & NA & - & - \\
\hline FTND scores & 6.12 & 1.06 & 0 & NA & - & - \\
\hline
\end{tabular}

Abbreviations: AUDIT, Alcohol Use Disorder Identification Test; BIS-I I, Barratt Impulsiveness Scale version I I; FTND, Fagerström Test for Nicotine Dependence; ISCED, International Standard Classification of Education; NA, not applicable; NART, National Adult Reading Test.

ACC was calculated in order to examine group differences in tissue composition. The ACC mask was defined by merging the individually placed spectroscopy voxel position in normalized space in order to correspond to the size and placement of the MRS voxel that was used for obtaining MRS spectra in the left dACC.

\section{Statistical Analyses}

All demographic and behavioural data analyses were carried out using SPSS 16.0 (SPSS, Chicago, IL). All data were normally distributed. Differences in baseline characteristics between groups were analysed using independent $t$-tests. A repeated measures ANOVA was conducted to assess the effect of NAC treatment on metabolite levels in the dACC between the two groups. Treatment (medication-free $v s$ NAC) was modeled as a within-subject factor and group (CD vs HC) was modeled as a between-subject factor. Administration order of NAC did not affect between-subject or within-subject differences in metabolite levels and was therefore not included as a covariate in the analyses. The post hoc tests were employed to examine significant differences between groups and within groups with and without NAC administration. Relationships between substance use, impulsivity measures, and Glu levels were explored using bivariate correlation and linear regression analyses. The significance criterion was set to $p<0.05$.
Table 2 NAC-Induced Changes in Glutamate (Glu), NAcetylaspartate (NAA), and Glutamate+Glutamine (Glx) Referenced to Creatine and Referenced to the Unsuppressed Water Signal

\begin{tabular}{|c|c|c|c|c|c|}
\hline \multirow[t]{2}{*}{ Metabolite } & \multirow[t]{2}{*}{ Session } & CD group & HC group & \multirow[t]{2}{*}{$F(d f)^{a}$} & \multirow[t]{2}{*}{$P$-value } \\
\hline & & \multicolumn{2}{|c|}{ Mean (SE) } & & \\
\hline \multicolumn{6}{|c|}{ Referenced to creatine } \\
\hline \multirow[t]{2}{*}{ Glu } & Medication-free & $1.49(0.07)$ & $1.32(0.04)$ & $5.46(1,20)$ & 0.03 \\
\hline & NAC & $1.32(0.04)$ & $1.35(0.05)$ & & \\
\hline \multirow[t]{2}{*}{ Glx } & Medication-free & $2.01(0.08)$ & $1.91(0.06)$ & $3.69(1,20)$ & 0.07 \\
\hline & NAC & $1.94(0.06)$ & $2.00(0.07)$ & & \\
\hline \multirow[t]{2}{*}{ NAA } & Medication-free & $1.24(0.04)$ & $1.29(0.05)$ & $0.03(1,20)$ & 0.87 \\
\hline & NAC & $1.21(0.03)$ & $1.25(0.04)$ & & \\
\hline \multicolumn{6}{|c|}{ Referenced to the unsuppressed water signal } \\
\hline \multirow[t]{2}{*}{ Glu } & Medication-free & $8.39(0.27)$ & $7.36(0.23)$ & $4.90(1,20)$ & 0.04 \\
\hline & NAC & $7.69(0.25)$ & $7.59(0.21)$ & & \\
\hline \multirow[t]{2}{*}{ Glx } & Medication-free & II.33 (0.36) & $0.57(0.29)$ & $2.51(1,20)$ & 0.13 \\
\hline & NAC & II.15 (0.37) & I I.45 (0.37) & & \\
\hline \multirow[t]{2}{*}{ NAA } & Medication-free & $6.99(0.16)$ & $7.12(0.12)$ & $0.19(1,20)$ & 0.66 \\
\hline & NAC & $6.95(0.08)$ & $6.99(0.18)$ & & \\
\hline \multirow[t]{2}{*}{$\mathrm{Cr}$} & Medication-free & $5.66(0.14)$ & $5.60(0.16)$ & $0.01(1,20)$ & 0.92 \\
\hline & NAC & $5.75(0.11)$ & $5.72(0.19)$ & & \\
\hline
\end{tabular}

${ }^{a}$ Results are presented for the treatment (medication-free $v s$ NAC) $\times$ group $(C D$ vs $H C$ ) interaction effect. There were no significant main effects of session or group.

\section{RESULTS}

\section{Sample Characteristics}

The demographic, clinical, and substance use characteristics are presented in Table 1 . Out of 10 CD subjects, 2 tested positive for cocaine and were excluded from further analyses. The CD group did not differ from the HC group with regard to age, educational level, and IQ. The CD group had significantly higher impulsivity scores measured by the BIS-11. HC subjects did not report any cocaine use or other drug use and consumed significantly less alcohol compared with CD subjects. No adverse events were reported in the study.

\section{Main Outcome: Effect of NAC on Glu Levels}

No differences between groups were found, both in the medication-free and NAC condition, in the gray matter, white matter, and cerebrospinal fluid content of the dACC region corresponding to the ${ }^{1} \mathrm{H}$ MRS voxel (all $p$-values $>0.3$ ) and were therefore not included as covariates in subsequent analyses. A repeated measures ANOVA revealed no main effect for group $(\mathrm{F}(1,20)=1.68, p=0.21)$ or treatment $(\mathrm{F}(1,20)=2.21, p=0.15)$ on dACC Glu relative to creatine $(\mathrm{Glu} / \mathrm{Cr})$. However, a significant interaction between group and treatment was present (see Table 2). The post hoc tests revealed that there was a significant reduction in $\mathrm{Glu} / \mathrm{Cr}$ in the NAC condition compared with the medication-free condition in the CD group $(t(7)=3.08$, 
$p=0.02)$, whereas NAC had no effect on $\mathrm{Glu} / \mathrm{Cr}$ in the $\mathrm{HC}$ group $(t(13)=-0.64, p=0.53)$. In the medication-free condition, significant higher $\mathrm{Glu} / \mathrm{Cr}$ in the $\mathrm{CD}$ group compared with the HC group was found $(t(20)=2.26$, $p=0.04)$. After administration of a single dose of NAC, differences in $\mathrm{Glu} / \mathrm{Cr}$ between the two groups disappeared $(t(20)=-0.24, p=0.81)$. These $\mathrm{Glu} / \mathrm{Cr}$ changes by NAC are graphically shown in Figure 2. Analysis with Glu concentrations referenced to the unsuppressed water signal revealed a similar group by treatment interaction (Table 2), which was driven by a significant higher Glu concentration in the medication-free condition in the $\mathrm{CD}$ group compared with the HC group $(t(20)=2.80, p=0.01)$ and a significant reduction in $\mathrm{Glu} / \mathrm{Cr}$ in the NAC condition compared with the medication-free condition only in the $\mathrm{CD}$ group $(t(7)=3.48, p=0.01)$.

Across groups, a regression analysis revealed that medication-free $\mathrm{Glu} / \mathrm{Cr}$ was predictive for the effect of NAC on Glu/ $\operatorname{Cr}(\beta=0.70, t(20)=4.43, p<0.001)$ and that it explained a significant and substantial proportion of variance in $\mathrm{Glu} / \mathrm{Cr}$ changes by NAC $\left(R^{2}=0.50, \mathrm{~F}(1,20)=19.58, p<0.001\right.$; see Figure 3a).

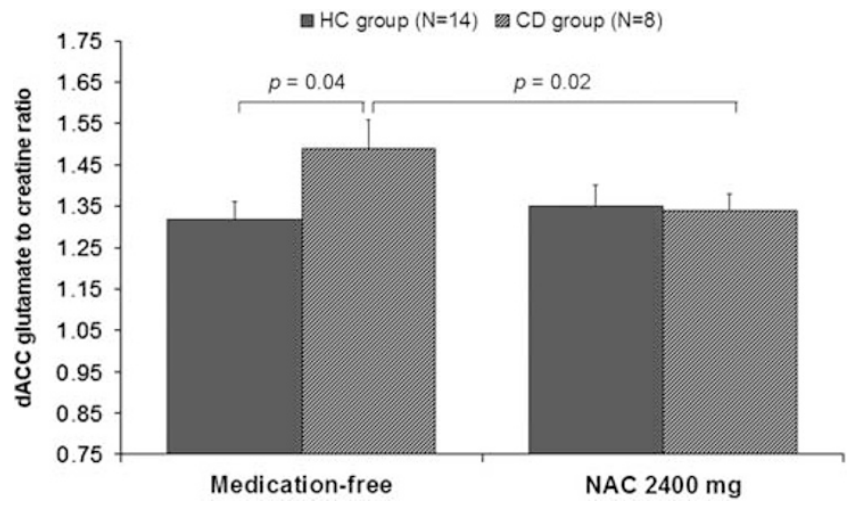

Figure 2 Effect of a single dose of NAC (2400 mg) on Glu/Cr in the left $\mathrm{dACC}$ in both $\mathrm{CD}$ and $\mathrm{HC}$ groups (mean $\pm \mathrm{SE}$ ). Medication-free $\mathrm{Glu} / \mathrm{Cr}$ was significantly higher in the $C D$ group when compared with the $\mathrm{HC}$ group. Administration of NAC reduced Glu/Cr in the CD group, whereas it had no effect on the $\mathrm{HC}$ group.
As NAC had an effect on Glu/Cr only in the CD group, an additional analysis was conducted within the $\mathrm{CD}$ group to test whether responders $v s$ nonresponders to the NAC challenge in terms of $\mathrm{Glu} / \mathrm{Cr}$ reduction differed in their medication-free Glu/Cr. A median split based on NACinduced $\mathrm{Glu} / \mathrm{Cr}$ changes revealed that responders had a significantly higher medication-free $\mathrm{Glu} / \mathrm{Cr}$ compared with nonresponders $(t(6)=2.90, p=0.03)$.

Because the measure of Glu was based on the ratio of Glu to creatine, we explored whether these results might have been caused by an effect of NAC on creatine. Creatine levels were unaffected by NAC treatment in both groups. In addition, NAC had no significant effect on other metabolite levels (Table 2).

\section{Impulsivity and Glu Levels}

Because of the involvement of the dACC in impulse control, we investigated whether $\mathrm{Glu} / \mathrm{Cr}$ within the dACC was related to general impulsivity across groups. There was a significant positive correlation between medication-free $\mathrm{Glu} / \mathrm{Cr}$ and impulsivity as measured by the BIS-11 (total impulsivity score, $r(22)=0.53, p=0.01$; cognitive impulsivity subscale, $r(22)=0.65, p<0.01$; nonplanning impulsivity subscale, $r(22)=0.48, p=0.02$, but not for the motor impulsivity subscale, $r(22)=0.26, p=0.25)$. In addition, a regression analysis revealed that higher BIS-11 total scores were predictive of NAC-induced decreases in $\mathrm{Glu} / \mathrm{Cr}(\beta=0.47$, $t(22)=2.38, p=0.03$; see Figure $3 \mathrm{~b})$. The total BIS-11 score explained a significant and substantial proportion of the variance in NAC-induced $\mathrm{Glu} / \mathrm{Cr}$ changes $\left(R^{2}=0.22\right.$, $\mathrm{F}(1,20)=5.66, p=0.03)$.

\section{Self-Reported Substance Use and Glu Levels}

Within the $C D$ group, there was a trend toward a negative correlation between self-reported total cocaine use during the 6 months before participation and medication-free Glu/ $\mathrm{Cr}$ in the dACC $(r(8)=-0.67, p=0.07)$. No associations with other cocaine use measures such as abstinence duration or other drug use such as number of cigarettes smoked and FTND scores in the CD group were found.

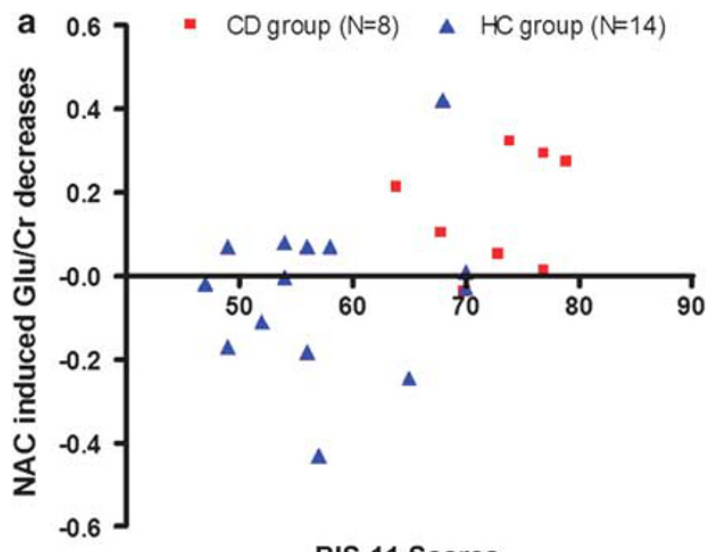

BIS-11 Scores

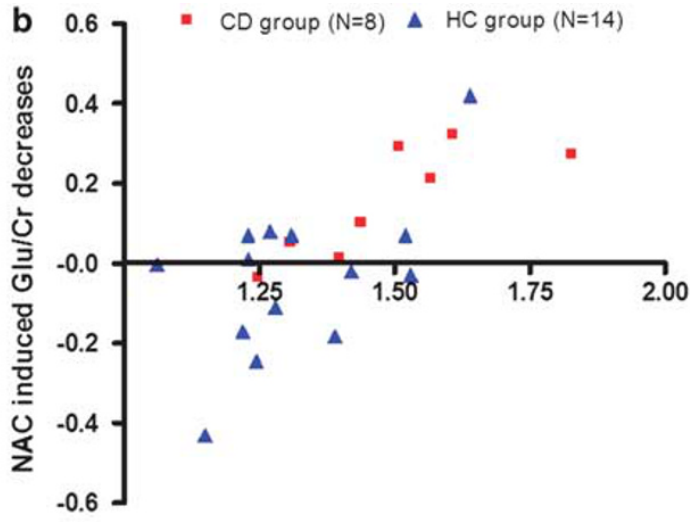

Medication-free $\mathrm{Glu} / \mathrm{Cr}$

Figure $3 \mathrm{NAC}$-induced $\mathrm{Glu} / \mathrm{Cr}$ decreases in the dACC were significantly predicted by (a) medication-free $\mathrm{Glu} / \mathrm{Cr}$ in the $\mathrm{dACC}\left(R^{2}=0.50, p<0.00 \mathrm{I}\right)$ and (b) trait impulsivity measured by the BIS-II total score $\left(R^{2}=0.22, p=0.03\right)$, across groups. 
Moreover, alcohol use and AUDIT scores did not correlate with $\mathrm{Glu} / \mathrm{Cr}$ in the $\mathrm{CD}$ group or in the $\mathrm{HC}$ group. Because both total cocaine use and BIS-11 impulsivity scores were correlated with medication-free $\mathrm{Glu} / \mathrm{Cr}$, we examined whether cocaine use and BIS-11 scores were also correlated. However, the BIS-11 total score and scores on the BIS-11 subscales were not correlated with total cocaine use.

\section{DISCUSSION}

Using ${ }^{1} \mathrm{H}$ MRS, the current study is the first to demonstrate a significant reduction in Glu/Cr in the left dACC by a single dose of NAC $(2400 \mathrm{mg})$ in cocaine-dependent patients, whereas NAC had no effect on $\mathrm{Glu} / \mathrm{Cr}$ in healthy controls. In the medication-free condition, significant higher $\mathrm{Glu} / \mathrm{Cr}$ was found in cocaine-dependent subjects compared with healthy controls, which normalized after a single administration of NAC. The current results are in line with preclinical studies indicating that NAC restores Glu abnormalities only when Glu homeostasis is disturbed, as for example by chronic exposure to cocaine (Baker et al, 2003b). Furthermore, higher $\mathrm{Glu} / \mathrm{Cr}$ at baseline was associated with general impulsivity ratings and both medication-free $\mathrm{Glu} / \mathrm{Cr}$ and impulsivity predicted NAC-induced changes in Glu/Cr.

These findings seem to be in contrast with those of Baker et al (2003b) showing lower levels of extracellular Glu in the nucleus accumbens in cocaine-treated rats compared with controls. In this rodent study, administration of NAC normalized basal levels of extracellular Glu. It should be noted, however, that ${ }^{1} \mathrm{H}$ MRS is not able to distinguish extracellular from intracellular Glu and primarily reflects the more abundant intracellular Glu, which is present in neuronal and glial metabolic and neurotransmitter pools (Gruetter et al, 1998). In the brain, basal levels of extracellular Glu are maintained by the cystine/glutamate antiporter (system $\mathrm{x}_{\mathrm{c}}^{-}$) exchanging extracellular cystine for intracellular Glu. The NAC-induced rise in extracellular Glu in cocaine-dependent rats has been attributed to restoration of system $\mathrm{x}_{\mathrm{c}}^{-}$functioning, which is predominantly expressed on glial cells (Baker et al, 2003b; Moussawi et al, 2011). Enhancement of the exchange of intracellular Glu for extracellular cystine by NAC would be expected to reduce glial intracellular Glu and, therefore, the current findings of increased medication-free levels of Glu and the NACinduced reduction of Glu in CD may stem from glial metabolic pools of Glu in the dACC. In addition, repeated cocaine administration has been associated with increased Glu neurotransmission in rats in medial prefrontal cortex by the reduced ability of mGluR2/3 receptors to inhibit synaptic Glu release (Xie and Steketee, 2008). Extracellular Glu stimulates presynaptic mGluR2/3 receptors and a NACinduced increase in extracellular Glu has been found to reduce neuronal Glu transmission by increased tonic activation of the mGluR2/3 receptors (Baker et al, 2003b; Moussawi et al, 2011). Our findings of NAC-induced Glu reduction may therefore also represent neurotransmitter pools in the dACC. Studies using carbon-13 spectroscopy, a technique that can differentiate between neurotransmitter and metabolic pools of Glu, may provide more insight into the source of the currently found Glu changes by NAC (Shen et al, 1999). Noteworthy is that quantification of Glu is difficult partly because it largely overlaps with glutamine in its chemical shift range, which leads to increased fitting errors. Although Glu was individually quantified separately from glutamine (Gln) and Glx with reasonable fitting errors (CRLBs all below 12\%) and represented $\sim 50-80 \%$ of the total Glx signal (Pouwels and Frahm, 1998), undetected contributions of other overlapping peaks such as Gln cannot be ruled out completely, especially considering the finding that NAC had no effect on Glx that largely consists of Glu. Detection of Gln alone by means of ${ }^{1} \mathrm{H}$ MRS is even more challenging and the current study did not allow reliable evaluation of Gln changes. Quantifying Gln (separately from Glu) could be of particular interest, as synaptic Glu taken up by glial cells is converted into glutamine before returning to the presynaptic neuron for conversion back into Glu (Magistretti and Pellerin, 1999) and therefore Gln may be a more accurate index of overall glutamatergic neurotransmission than Glu (Rothman et al, 2003). Future studies using more advanced spectral editing techniques such as a spectrally selective refocusing method (Choi et al, 2006) or 2D J-resolved spectroscopy (Jensen et al, 2009) for improved separation of Glu from Gln are required to further characterize the effect of NAC on the Glu system in the human brain.

The current finding of an increased medication-free $\mathrm{Glu} / \mathrm{Cr}$ in cocaine-dependent subjects relative to healthy controls is consistent with a recent study by Liu et al (2011) investigating the effects of chronic exposure to cocaine on $\mathrm{Glu} / \mathrm{Cr}$ in the putamen of squirrel monkeys using ${ }^{1} \mathrm{H}$ MRS. After 9 months, $\mathrm{Glu} / \mathrm{Cr}$ and $\mathrm{Gln} / \mathrm{Cr}$ were significantly higher in cocaine-treated monkeys compared with baseline levels and to $\mathrm{Glu} / \mathrm{Cr}$ in saline-treated monkeys. Higher ACC Glu/Cr was also found in young alcoholics (Lee et al, 2007). Moreover, treatment with acamprosate, a Glu-mediating compound, reduced ACC $\mathrm{Glu} / \mathrm{Cr}$ whereas $\mathrm{Glu} / \mathrm{Cr}$ was increased during a 4 -week placebo treatment in alcohol-dependent patients (Umhau et al, 2010). Although some of our CD subjects met the criteria for alcohol abuse, we did not find an association between $\mathrm{Glu} / \mathrm{Cr}$ and alcohol use. This could perhaps be explained by the amount of alcohol consumed in our CD group (mean of 3.5 drinks/day), which was not as high as reported in the study of Umhau et al (2010) (mean of $\sim 15$ drinks/day), whereas the study of Lee et al (2007) did not measure daily alcohol consumption. In contrast to findings of increased Glu levels in substance dependence, Yang et al (2009) found significantly lower levels of ACC Glu/Cr in chronic cocaine users, although they did measure Glu in a functionally different division of the ACC, namely the rACC as opposed to dACC in the current study. Whereas the dACC is more involved in cognitive processing, the rACC is mainly activated in response to emotional content (Bush et al, 2000). These distinct areas of the ACC have been found to be differentially affected by chronic cocaine use. Whereas hypoactivation has been observed in the dACC during response inhibition tasks and in response to drug cues (Hester and Garavan, 2004; Li et al, 2008; Volkow et al, 2011), hyperactivation (or diminished deactivation) has been detected in the rACC (Brodmann area 25) and adjacent ventromedial frontal areas especially in the presence of drug-related cues in cocaine-dependent patients (Kilts et al, 2001; Volkow et al, 2005). This interaction between a 
diminished functioning of a cognitive control brain network (including the $\mathrm{dACC}$ ) and an increased responsiveness to drug-cues in reward-processing areas (including the rACC) has been proposed to underlie compulsive drug taking (Baler and Volkow, 2006). In line with these findings of differentially affected subdivisions of the ACC, Yang et al (2009) found a positive correlation between years of cocaine use and $\mathrm{Glu} / \mathrm{Cr}$, that is, the longer the cocaine use, the higher the rACC Glu/Cr, whereas we found a trend towards a negative correlation between total cocaine use in the preceding 6 months before participation and dACC $\mathrm{Glu} / \mathrm{Cr}$ (unfortunately, we did not have a measure of years of cocaine use in the present study). However, both correlations seem to be in the opposite direction of what would have been expected. In line with the self-medication hypothesis of cocaine use, a possible explanation might be that these Glu abnormalities are a pre-existing risk factor for the development of addiction and are actually normalized with continued cocaine use. Clearly, the relationship between brain Glu levels and cocaine dependence is not straightforward but rather complex, depending on multiple facets of individual drug use patterns, and needs further investigation.

The dACC is a key region involved in impulse inhibition (Chambers et al, 2009) and maladaptive high levels of impulsivity have been associated with diminished ACC functioning in substance dependence (Forman et al, 2004; Kaufman et al, 2003; Lee et al, 2005; Li et al, 2008; Meade et al, 2011). Preclinical literature has indicated a role for Glu in impulsivity (for a review, see Pattij and Vanderschuren, 2008). For instance, selective and nonselective NMDA receptor antagonists have been shown to increase impulsive behavior in animal models (Higgins et al, 2003; Mirjana et al, 2004). Systemic pretreatment with an mGlu2/3 receptor agonist attenuates impulsive behavior seen after serotonin receptor stimulation (Wischhof et al, 2011). In humans, a recent study of Hoerst et al (2010) examined Glu levels in the dACC in patients with borderline personality disorder and healthy controls. Irrespective of diagnosis, higher Glu/Cr was associated with higher BIS-11 total scores and cognitive impulsivity subscale scores (Hoerst et al, 2010). Anterior cingulate $\mathrm{Glu} / \mathrm{Cr}$ was also found to be increased in untreated children with ADHD, a disorder characterised by impaired impulse control (Hammerness et al, 2012). In keeping with these findings, the current study revealed that medication-free Glu/Cr in the dACC was associated with (cognitive) impulsivity measured by the BIS-11. These results suggest that Glu abnormalities underlie impaired (left) dACC functioning associated with high levels of impulsivity found in substance dependence. Preclinical studies have reported that NAC treatment prevents reinstatement of drug-seeking behavior in cocaine-treated rats by restoring Glu homeostasis and thereby increasing tonic activation of the mGluR $2 / 3$ receptors (Baker et al, 2003b). In humans, NAC reduces the desire to use cocaine in the presence of cocaine-related cues in cocaine-dependent patients (LaRowe et al, 2007). Impulsivity is an important predictor of relapse into substance abuse (Bowden-Jones et al, 2005; Brewer et al, 2008; Goudriaan et al, 2008; Krishnan-Sarin et al, 2007; MacKillop and Kahler, 2009; Moeller et al, 2001). In the current study we found that impulsivity ratings predict NAC-induced $\mathrm{Glu} / \mathrm{Cr}$ changes. Because impulsive behavior is in part regulated by mGlu2/3 receptor activation (Wischhof et al, 2011), and NAC increases mGluR $2 / 3$ activation resulting in prevention of cue-induced reinstatement of cocaine-seeking behavior (Baker et al, 2003b), one may speculate that impulsivity mediates the relation between NAC-induced Glu changes and reductions in cue-induced craving and prevention of cue-induced reinstatement by NAC treatment. However, because we did not include measures for NAC-induced changes in impulsivity and craving, future research is needed to further delineate the interrelation between Glu, impulsivity, and craving for cocaine or relapse into cocaine abuse.

As the current results were obtained in a pilot study, our results should be viewed in light of some methodological limitations. First, the current study was an open-label study, and hence we cannot rule out the possibility that subjective effects interacted with changes in Glu levels. However, we failed to observe order effects (NAC treatment $v s$ no treatment) and it seems rather unlikely that expectancies have such a profound effect on Glu levels in the left dACC. Therefore, we feel that the open-label aspect of the study is not a serious threat to the validity of our findings. Second, the sample size was modest, especially with regard to the $C D$ group. The results were similar when two more $\mathrm{CD}$ subjects who tested positive for cocaine were included (data not shown). However, future studies including larger sample sizes are warranted to replicate the current findings. Third, the current study was designed as a first step toward investigating the effects of NAC on Glu levels in the human brain, and for this purpose we used a single dose of NAC based on the findings of Moran et al (2005) showing that pretreatment with a single dose of NAC prevents relapse in cocaine-seeking behavior in an animal model of reinstatement. However, future research examining the effects of longer treatment with NAC and dose effects of NAC on Glu levels is needed to confirm the current findings. Moreover, because no clinical measures were included in the current study and the impulsivity questionnaire was only administered once, the implications of the observed NAC-induced Glu changes for clinical outcome and impulsivity remain to be elucidated. Double-blind, placebo-controlled studies implementing longer treatment durations are required to further clarify the effects of NAC on the brain Glu system and their consequences on clinical measures and state impulsivity. Another limitation is that some of our cocainedependent sample had secondary alcohol problems and most of them were smokers. Therefore, we cannot rule out effects of alcohol use and smoking on the current findings of the NAC-induced reduction in Glu levels. However, we did show that there was no correlation between NACinduced Glu changes and baseline alcohol use and smoking characteristics such as alcohol consumption in the past 6 months, AUDIT scores, number of cigarettes smoked per week, and FTND scores. Finally, drawing conclusions regarding the specificity of our findings are limited by the fact that we did not acquire metabolite data from other regions than the left dACC. We chose the left dACC as our region of interest because most Glu abnormalities in previous human studies were located in the ACC. Given the findings of animal studies with NAC, it would be of particular interest to also investigate the effects of NAC on 
Glu levels in the nucleus accumbens. It is, however, difficult to reliably evaluate Glu levels in this particular region because of field inhomogeneities, but future ${ }^{1} \mathrm{H}$ MRS studies performed at a higher field strength (eg, 7 Tesla) or with access to more advanced spectral editing techniques should be able to establish whether the nucleus accumbens is similarly or differently affected.

Notwithstanding these limitations, we believe that the current ${ }^{1} \mathrm{H}$ MRS study is an important step toward unraveling the mechanisms by which NAC acts on the Glu system in substance-dependent patients. NAC has been proven to successfully prevent relapse into cocaine-seeking behavior by restoring Glu homeostasis in animal models of reinstatement. Our study is the first to demonstrate a similar effect of NAC on brain Glu abnormalities in cocainedependent humans. Together with treatment studies indicating that NAC has a beneficial effect in patients with cocaine dependence (LaRowe et al, 2007; Mardikian et al, 2007), pathological gambling (Grant et al, 2007), marijuana users (Gray et al, 2010), and nicotine dependence (Knackstedt et al, 2009; Schmaal et al, 2011), the current study suggests that this effect might in part be mediated by the ability of NAC to normalize glutamatergic abnormalities. In addition, the current study demonstrates that baseline Glu/ $\mathrm{Cr}$ and baseline (cognitive) impulsivity predict the ability of NAC to normalize Glu abnormalities. This is consistent with the preclinical literature and current observations of a lack of effect of NAC in controls with normal Glu levels. These findings also suggest that NAC might be especially effective in patients with high dACC Glu levels (according to a pretreatment ${ }^{1} \mathrm{H}$ MRS scan) and/or patients with high levels of self-reported (cognitive) impulsivity. However, future studies are required to determine the most predictive cutoff point of ${ }^{1} \mathrm{H}$ MRS-based Glu levels or BIS-11 impulsivity.

In conclusion, ${ }^{1} \mathrm{H}$ MRS is a valuable noninvasive tool to study Glu system functioning in cocaine dependence and to detect changes induced by Glu modulating compounds such as NAC. The current pilot study shows preliminary evidence for the ability of NAC to normalize Glu homeostasis in cocaine-dependent patients and provides a neurobiochemical rationale for future trials with NAC as a treatment for cocaine dependence. Furthermore, baseline Glu levels were predictive of NAC-induced Glu changes, suggesting that ${ }^{1} \mathrm{H}$ MRS may serve as a biological marker to predict treatment outcome.

\section{ACKNOWLEDGEMENTS}

We thank The Netherlands Organization for Scientific Research for their financial support (ZonMW Grant 31160003).

\section{DISCLOSURE}

The authors declare no conflict of interest.

\section{REFERENCES}

American Psychiatry Association (1994). Diagnostic and Statistical Manual of Mental Disorders. American Psychiatric Press: Washington DC.
Ashburner J, Friston KJ (2005). Unified segmentation. Neuroimage 26: 839-851.

Babor TF, Kranzler HR, Lauerman RJ (1989). Early detection of harmful alcohol consumption: comparison of clinical, laboratory, and self-report screening procedures. Addict Behav 14: 139-157.

Baker DA, McFarland K, Lake RW, Shen H, Tang XC, Toda S et al (2003a). Neuroadaptations in cystine-glutamate exchange underlie cocaine relapse. Nat Neurosci 6: 743-749.

Baker DA, McFarland K, Lake RW, Shen H, Toda S, Kalivas PW (2003b). N-Acetyl Cysteine-Induced Blockade of Cocaine-Induced Reinstatement. New York ACAD Sciences: New York 351 pp.

Baler RD, Volkow ND (2006). Drug addiction: the neurobiology of disrupted self-control. Trends Mol Med 12: 559-566.

Bowden-Jones $\mathrm{H}$, McPhillips $\mathrm{M}$, Rogers R, Hutton S, Joyce E (2005). Risk-taking on tests sensitive to ventromedial prefrontal cortex dysfunction predicts early relapse in alcohol dependency: a pilot study. J Neuropsychiatry Clin Neurosci 17: 417-420.

Brewer JA, Worhunsky PD, Carroll KM, Rounsaville BJ, Potenza MN (2008). Pretreatment brain activation during stroop task is associated with outcomes in cocaine-dependent patients. Biol Psychiatry 64: 998-1004.

Bush G, Luu P, Posner MI (2000). Cognitive and emotional influences in anterior cingulate cortex. Trends Cogn Sci 4: 215-222.

Chambers CD, Garavan H, Bellgrove MA (2009). Insights into the neural basis of response inhibition from cognitive and clinical neuroscience. Neurosci Biobehav Rev 33: 631-646.

Chang L, Mehringer CM, Ernst T, Melchor R, Myers H, Forney D et al (1997). Neurochemical alterations in asymptomatic abstinent cocaine users: a proton magnetic resonance spectroscopy study. Biol Psychiatry 42: 1105-1114.

Choi C, Coupland NJ, Bhardwaj PP, Malykhin N, Gheorghiu D, Allen PS (2006). Measurement of brain glutamate and glutamine by spectrally-selective refocusing at 3 Tesla. Magn Reson Med 55: 997-1005.

Ernst T, Chang L (2008). Adaptation of brain glutamate plus glutamine during abstinence from chronic methamphetamine use. J Neuroimmune Pharmacol 3: 165-172.

Forman SD, Dougherty GG, Casey BJ, Siegle GJ, Braver TS, Barch $\mathrm{DM}$ et al (2004). Opiate addicts lack error-dependent activation of rostral anterior cingulate. Biol Psychiatry 55: 531-537.

Gallinat J, Schubert F (2007). Regional cerebral glutamate concentrations and chronic tobacco consumption. Pharmacopsychiatry 40: 64-67.

Garavan H, Hester R (2007). The role of cognitive control in cocaine dependence. Neuropsychol Rev 17: 337-345.

Goldstein RZ, Alia-Klein N, Tomasi D, Carrillo JH, Maloney T, Woicik PA et al (2009). Anterior cingulate cortex hypoactivations to an emotionally salient task in cocaine addiction. Proc Natl Acad Sci USA 106: 9453-9458.

Goudriaan AE, Oosterlaan J, De BE, Van Den Brink W (2008). The role of self-reported impulsivity and reward sensitivity versus neurocognitive measures of disinhibition and decision-making in the prediction of relapse in pathological gamblers. Psychol Med 38: 41-50.

Grant JE, Kim SW, Odlaug BL (2007). N-acetyl cysteine, a glutamate-modulating agent, in the treatment of pathological gambling: a pilot study. Biol Psychiatry 62: 652-657.

Gray KM, Watson NL, Carpenter MJ, LaRowe SD (2010). Nacetylcysteine (NAC) in young marijuana users: an open-label pilot study. Am J Addict 19: 187-189.

Gruetter R, Seaquist ER, Kim S, Ugurbil K (1998). Localized in vivo 13C-NMR of glutamate metabolism in the human brain: initial results at 4 tesla. Dev Neurosci 20: 380-388.

Hammerness P, Biederman J, Petty C, Henin A, Moore CM (2012). Brain biochemical effects of methylphenidate treatment using proton magnetic spectroscopy in youth with attention-deficit 
hyperactivity disorder: a controlled pilot study. CNS Neurosci Ther 18: $34-40$.

Heatherton TF, Kozlowski LT, Frecker RC, Fagerstrom KO (1991). The Fagerstrom Test for Nicotine Dependence: a revision of the Fagerstrom Tolerance Questionnaire. Br J Addict 86: 1119-1127.

Hester R, Garavan H (2004). Executive dysfunction in cocaine addiction: evidence for discordant frontal, cingulate, and cerebellar activity. J Neurosci 24: 11017-11022.

Higgins GA, Ballard TM, Huwyler J, Kemp JA, Gill R (2003). Evaluation of the NR2B-selective NMDA receptor antagonist Ro 63-1908 on rodent behaviour: evidence for an involvement of NR2B NMDA receptors in response inhibition. Neuropharmacology 44: 324-341.

Hoerst M, Weber-Fahr W, Tunc-Skarka N, Ruf M, Bohus M, Schmahl C et al (2010). Correlation of glutamate levels in the anterior cingulate cortex with self-reported impulsivity in patients with borderline personality disorder and healthy controls. Arch Gen Psychiatry 67: 946-954.

Holdiness MR (1991). Clinical pharmacokinetics of N-acetylcysteine. Clin Pharmacokinet 20: 123-134.

Jensen JE, Licata SC, Ongur D, Friedman SD, Prescot AP, Henry ME et al (2009). Quantification of J-resolved proton spectra in two-dimensions with LCModel using GAMMA-simulated basis sets at 4 Tesla. NMR Biomed 22: 762-769.

Kaufman JN, Ross TJ, Stein EA, Garavan H (2003). Cingulate hypoactivity in cocaine users during a GO-NOGO task as revealed by event-related functional magnetic resonance imaging. J Neurosci 23: 7839-7843.

Kelley AE (2004). Memory and addiction: shared neural circuitry and molecular mechanisms. Neuron 44: 161-179.

Kilts CD, Schweitzer JB, Quinn CK, Gross RE, Faber TL, Muhammad F et al (2001). Neural activity related to drug craving in cocaine addiction. Arch Gen Psychiatry 58: 334-341.

Knackstedt LA, LaRowe S, Mardikian P, Malcolm R, Upadhyaya H, Hedden $S$ et al (2009). The role of cystine-glutamate exchange in nicotine dependence in rats and humans. Biol Psychiatry 65: 841-845.

Krishnan-Sarin S, Reynolds B, Duhig AM, Smith A, Liss T, McFetridge A et al (2007). Behavioral impulsivity predicts treatment outcome in a smoking cessation program for adolescent smokers. Drug Alcohol Depend 88: 79-82.

LaRowe SD, Myrick H, Hedden S, Mardikian P, Saladin M, McRae A et al (2007). Is cocaine desire reduced by N-acetylcysteine? Am J Psychiatry 164: 1115-1117.

Lee E, Jang DP, Kim JJ, An SK, Park S, Kim IY et al (2007). Alteration of brain metabolites in young alcoholics without structural changes. Neuroreport 18: 1511-1514.

Lee TM, Zhou WH, Luo XJ, Yuen KS, Ruan XZ, Weng XC (2005). Neural activity associated with cognitive regulation in heroin users: a fMRI study. Neurosci Lett 382: 211-216.

Li CS, Huang C, Yan P, Bhagwagar Z, Milivojevic V, Sinha R (2008). Neural correlates of impulse control during stop signal inhibition in cocaine-dependent men. Neuropsychopharmacology 33: $1798-1806$.

Liu X, Jensen JE, Gillis TE, Zuo CS, Prescot AP, Brimson M et al (2011). Chronic cocaine exposure induces putamen glutamate and glutamine metabolite abnormalities in squirrel monkeys. Psychopharmacology (Berl) 217: 367-375.

MacKillop J, Kahler CW (2009). Delayed reward discounting predicts treatment response for heavy drinkers receiving smoking cessation treatment. Drug Alcohol Depend 104: 197-203.

Madayag A, Lobner D, Kau KS, Mantsch JR, Abdulhameed O, Hearing $\mathrm{M}$ et al (2007). Repeated $\mathrm{N}$-acetylcysteine administration alters plasticity-dependent effects of cocaine. J Neurosci 27: 13968-13976.

Magistretti PJ, Pellerin L (1999). Astrocytes couple synaptic activity to glucose utilization in the brain. News Physiol Sci 14: $177-182$.
Mardikian PN, LaRowe SD, Hedden S, Kalivas PW, Malcolm RJ (2007). An open-label trial of $\mathrm{N}$-acetylcysteine for the treatment of cocaine dependence: a pilot study. Prog Neuropsychopharmacol Biol Psychiatry 31: 389-394.

McFarland K, Lapish CC, Kalivas PW (2003). Prefrontal glutamate release into the core of the nucleus accumbens mediates cocaineinduced reinstatement of drug-seeking behavior. J Neurosci 23: 3531-3537.

Meade CS, Lowen SB, MacLean RR, Key MD, Lukas SE (2011). fMRI brain activation during a delay discounting task in HIVpositive adults with and without cocaine dependence. Psychiatry Res 192: 167-175.

Mirjana C, Baviera M, Invernizzi RW, Balducci C (2004). The serotonin 5-HT2A receptors antagonist M100907 prevents impairment in attentional performance by NMDA receptor blockade in the rat prefrontal cortex. Neuropsychopharmacology 29: 1637-1647.

Moeller FG, Dougherty DM, Barratt ES, Schmitz JM, Swann AC, Grabowski J (2001). The impact of impulsivity on cocaine use and retention in treatment. J Subst Abuse Treat 21: 193-198.

Moran MM, McFarland K, Melendez RI, Kalivas PW, Seamans JK (2005). Cystine/glutamate exchange regulates metabotropic glutamate receptor presynaptic inhibition of excitatory transmission and vulnerability to cocaine seeking. J Neurosci 25: 6389-6393.

Moussawi K, Zhou W, Shen H, Reichel CM, See RE, Carr DB et al (2011). Reversing cocaine-induced synaptic potentiation provides enduring protection from relapse. Proc Natl Acad Sci USA 108: 385-390.

Mullins PG, Chen H, Xu J, Caprihan A, Gasparovic C (2008). Comparative reliability of proton spectroscopy techniques designed to improve detection of J-coupled metabolites. Magn Reson Med 60: 964-969.

Pattij T, Vanderschuren LJ (2008). The neuropharmacology of impulsive behaviour. Trends Pharmacol Sci 29: 192-199.

Patton JH, Stanford MS, Barratt ES (1995). Factor structure of the Barratt Impulsiveness Scale. J Clin Psychol 51: 768-774.

Pouwels PJ, Frahm J (1998). Regional metabolite concentrations in human brain as determined by quantitative localized proton MRS. Magn Reson Med 39: 53-60.

Provencher SW (1993). Estimation of metabolite concentrations from localized in vivo proton NMR spectra. Magn Reson Med 30: 672-679.

Reissner KJ, Kalivas PW (2010). Using glutamate homeostasis as a target for treating addictive disorders. Behav Pharmacol 21: 514-522.

Rothman DL, Behar KL, Hyder F, Shulman RG (2003). In vivo NMR studies of the glutamate neurotransmitter flux and neuroenergetics: implications for brain function. Annu Rev Physiol 65: 401-427.

Schmaal L, Berk L, Hulstijn KP, Cousijn J, Wiers RW, Van Den Brink W (2011). Efficacy of N-acetylcysteine in the treatment of nicotine dependence: a double-blind placebo-controlled pilot study. Eur Addict Res 17: 211-216.

Schmand B, Bakker D, Saan R, Louman J (1991). [The Dutch reading test for adults: a measure of premorbid intelligence level]. Tijdschr Gerontol Geriatr 22: 15-19.

Sheehan DV, Lecrubier Y, Sheehan KH, Amorim P, Janavs J, Weiller E et al (1998). The Mini-International Neuropsychiatric Interview (M.I.N.I.): the development and validation of a structured diagnostic psychiatric interview for DSM-IV and ICD-1. J Clin Psychiatry 59(Suppl 20): 22-33.

Shen J, Petersen KF, Behar KL, Brown P, Nixon TW, Mason GF et al (1999). Determination of the rate of the glutamate/ glutamine cycle in the human brain by in vivo 13C NMR. Proc Natl Acad Sci USA 96: 8235-8240.

Sobell LC, Sobell MB (1992). Timeline followback: a technique for assessing self-reported alcohol consumption. In: Litten RZ, 
NAC normalizes Glu levels in cocaine-dependent patients L Schmaal et al

Allen J (eds) Measuring Alcohol Consumption: Psychosocial and Biological Methods. Humana Press: New Jersey. pp 41-72.

Sun W, Rebec GV (2006). Repeated cocaine self-administration alters processing of cocaine-related information in rat prefrontal cortex. J Neurosci 26: 8004-8008.

Thoma R, Mullins P, Ruhl D, Monnig M, Yeo RA, Caprihan A et al (2011). Perturbation of the glutamate-glutamine system in alcohol dependence and remission. Neuropsychopharmacology 36: $1359-1365$.

Umhau JC, Momenan R, Schwandt ML, Singley E, Lifshitz M, Doty $\mathrm{L}$ et al (2010). Effect of acamprosate on magnetic resonance spectroscopy measures of central glutamate in detoxified alcohol-dependent individuals: a randomized controlled experimental medicine study. Arch Gen Psychiatry 67: 1069-1077.

Volkow ND, Tomasi D, Wang GJ, Fowler JS, Telang F, Goldstein $\mathrm{RZ}$ et al (2011). Reduced metabolism in brain "control networks" following cocaine-cues exposure in female cocaine abusers. PLoS One 6: e16573.

Volkow ND, Wang GJ, Ma Y, Fowler JS, Wong C, Ding YS et al (2005). Activation of orbital and medial prefrontal cortex by methylphenidate in cocaine-addicted subjects but not in controls: relevance to addiction. J Neurosci 25: 3932-3939.

Wischhof L, Hollensteiner KJ, Koch M (2011). Impulsive behaviour in rats induced by intracortical DOI infusions is antagonized by co-administration of an mGlu2/3 receptor agonist. Behav Pharmacol 22: 805-813.

Xie X, Steketee JD (2008). Repeated exposure to cocaine alters the modulation of mesocorticolimbic glutamate transmission by medial prefrontal cortex Group II metabotropic glutamate receptors. J Neurochem 107: 186-196.

Yang S, Salmeron BJ, Ross TJ, Xi ZX, Stein EA, Yang Y (2009). Lower glutamate levels in rostral anterior cingulate of chronic cocaine users - A (1)H-MRS study using TE-averaged PRESS at $3 \mathrm{~T}$ with an optimized quantification strategy. Psychiatry Res 174: 171-176.

Yucel M, Lubman DI, Harrison BJ, Fornito A, Allen NB, Wellard $\mathrm{RM}$ et al (2007). A combined spectroscopic and functional MRI investigation of the dorsal anterior cingulate region in opiate addiction. Mol Psychiatry 12: 691-702.

Zhou W, Kalivas PW (2008). N-acetylcysteine reduces extinction responding and induces enduring reductions in cue- and heroininduced drug-seeking. Biol Psychiatry 63: 338-340. 